neonatal characteristics (OR 1.97; CI [1.30-2.98]). In contrast, the smoking-related increased risk of apnea was no longer significant after adjustment for gestational age.

Conclusion: Prenatal nicotine (snuff) is associated with a higher risk of neonatal apnea than smoking. This implies that it is not safe to recommend nicotine replacement therapy (NRT) for smoking cessation during pregnancy.

162

\section{LONG-TERM CARDIO-RESPIRATORY REGULATION IN CHILDREN WITH RETT SYNDROME}

M. Rohdin 1 , M. Eriksson², M. Albåge², H. Lagercrantz ${ }^{1}$, M. Katz-Salamon ${ }^{1}$

${ }^{1}$ Woman and Child Health, Karolinska Institutet, ${ }^{2}$ Department of Neuropediatrics, Karolinska University Hospital, Stockholm, Sweden

Background: The cardiac and respiratory function requires an intricate coordination of afferent/efferent signals at several levels to secure homeostasis. Rett syndrome is characterised by a functional disintegration of physiological functions. Autonomic function in Rett syndrome has been studied mainly during short time spans (minutes-1 hour). In order to capture the full complexity of the autonomic function - a long-term study of the cardio-respiratory regulation was performed.

Methods: Patients with Rett syndrome in the Stockholm area were studied during one week in their home environment $(n=15)$. Heart rate and breathing were recorded via standard three-lead ECG electrodes. The depth and frequency of respiratory movements were measured via changes in impedance. Oxygenation was measured by pulse oxymetry. Care takers were asked to fill out a form during the monitoring enabling us to analyze the correlation between behaviour and cardiorespiratory reactions.

Results: All subjects revealed respiratory dysfunction and a majority had bradycardia and/ or tachycardia. In accordance with our previous findings, disturbances in cardio-respiratory function were present both when awake and asleep. Interestingly, there were pronounced variations in cardio-respiratory regulation from day to day and even from hour to hour in an individual patient. Furthermore, we also observed variations in cardio-respiratory events between individuals and situations.
Conclusions: Cardio-respiratory function in children with Rett syndrome is characterised by dramatic instability during wakefulness and sleep and differ among individuals. Cardio-respiratory regulation varies from hour to hour and between different situations in the individual Rett girl. Therefore, a long-term monitoring is decisive for adequate diagnostics and treatment.

163

HEALTH STATUS OF CHILDREN ADOPTED FROM KAZAKHSTAN IN BELGIUM

\author{
E. Peeters ${ }^{1}$, M. Meeus ${ }^{1}$, J. Ramet ${ }^{1}$,
} A. Van Gompel ${ }^{2}$, M. Wojciechowski ${ }^{1,2}$

${ }^{1}$ Department of Pediatrics, UZA Antwerp University Hospital, ${ }^{2}$ Institute of Tropical Medicine, Antwerp, Belgium

Objectives: evaluate the demographic characteristics and health status of children adopted from Kazakhstan at arrival in Belgium.

Material and methods: Retrospective review of data gathered at the Institute of Tropical Medicine (ITM).

Results: 17 children were seen in the ITM between from 01-2008 to 11-2009. Reason for adoption: $58 \%$ renunciation, $24 \%$ abandoned, $12 \%$ foundlings, $6 \%$ true orphans. All children lived in an orphanage prior to adoption. $29 \%$ were 'special needs' children. Age at arrival : 0,17 and 73 months; in $29 \%$ the birth date was uncertain.

Height z-scores and weight z-scores ranged from $-4,38$ to 0,82 (mean $-2,02$ ) and from $-3,34$ to 1,07 (mean $-0,98$ ). $47 \%$ of the children were stunted, $12 \%$ wasted.

The immunization status was complete in $42 \%$ and unknown in $29 \%$ of the cases.

Feces examination revealed Ascaris lumbricoides in $1 / 17$ \& Opisthorchiasis in $1 / 17$. $3 / 7$ of the children had serological evidence of Strongyloides stercoralis infection; thus far this has not been described in Kazakh children.

$47 \%$ of the adoptees were in good health at initial examination. Dysmorphic features and malformations were present in $29 \%$.

Conclusion: a considerable number of children had malformations, parasitic infections, or other problems. Stunted growth was present in a striking 